\title{
Biological activity of lipids extracted from two isolates of Fusarium oxysporum (environmental and clinical) in Galleria mellonella
}

\author{
Jessica Sepúlveda-Rivera ${ }^{a, c^{*}}$, Pedronel Araque Marín ${ }^{b}$, Carlos Peláez \\ Jaramilloa, Jose Miguel Acevedo Ra $^{a}$, Diana Yuledy Molina $\mathbf{C}^{c}$, \\ Maria del Pilar Jiménez Alzatec \\ jiessica.sepulveda@udea.edu.co
}

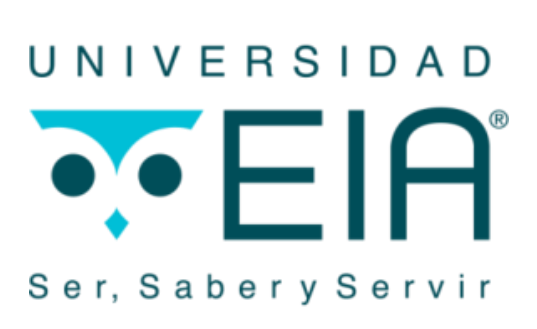

aGrupo Interdisciplinario de Estudios Moleculares-GIEM, Faculty of Exact and Natural Sciences, Universidad de Antioquia.

bGrupo de Investigación e Innovación en Formulaciones Químicas, Basic Science Department, Universidad EIA.

'Grupo de Micología Médica, Faculty of Medicine, Microbiology and Parasitology Department, Universidad de Antioquia.

\section{INTRODUCTION}

Fusarium oxysporum (Hypocreales: Nectriaceae) is a pathogen trans-kingdom species complex. The lipids role in $F$. oxysporum pathogenesis is poorly understood but these macromolecules are important for fungal development and virulence [1]. We evaluate the virulence of conidia, the chemical composition and the biological activity of lipids extracted from two $F$. oxysporum isolates (environmental and clinical) in the G. mellonella invertebrate model.

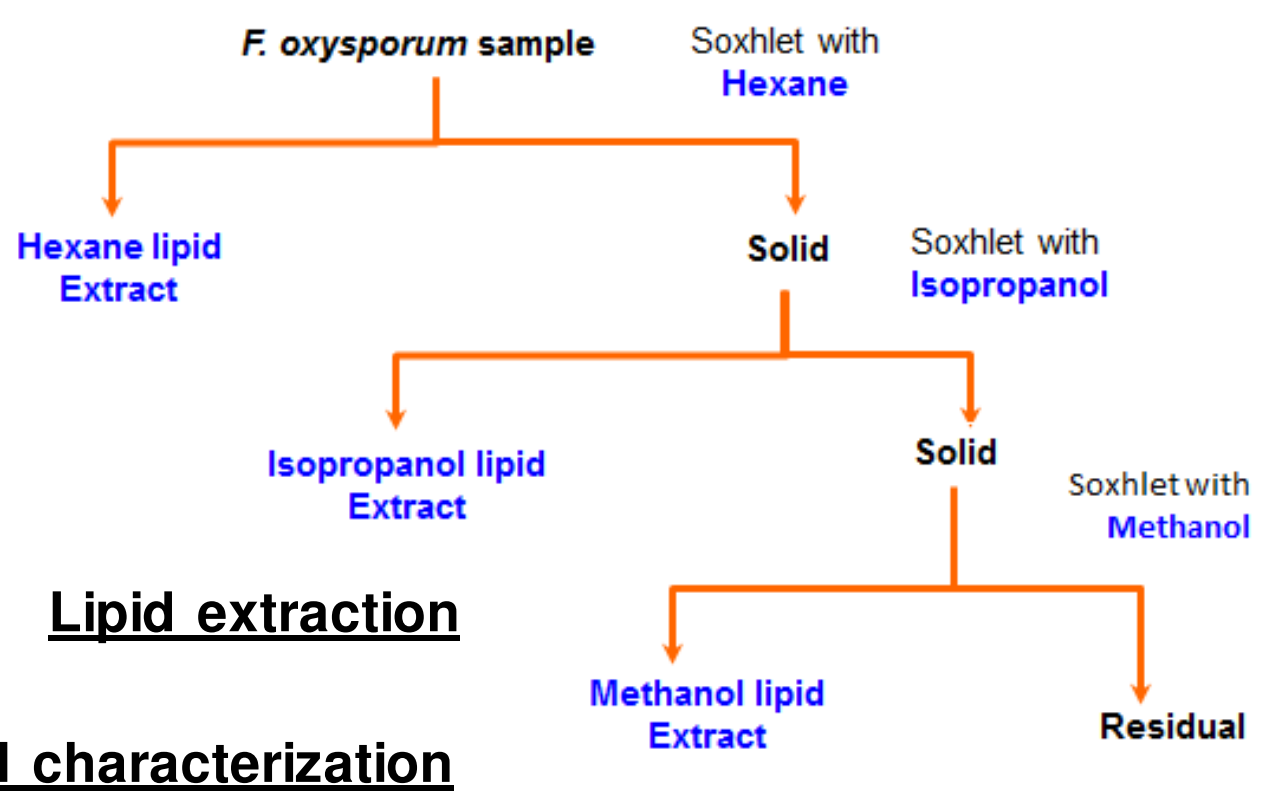

Lipid characterization

Mono and bidimensional TLC, HPLC-MS, FAMES

Biological assays

F. oxysporum (environmental and clinical) isolates CONIDIA

$10^{4}, 10^{5}, 10^{6}$

LIPIDS

$10^{2}, 10^{3}$

$10^{4} \mu \mathrm{g} / \mathrm{mL}$

CO-STIMULUS

Sub lethal

$10^{7}$ conidia/mL

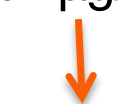

G. mellonella caterpillars injection

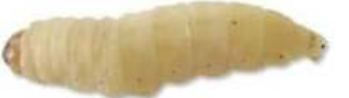

\section{RESULTS}

1. Conidia from $F$. oxysporum environmental isolate are more virulent than conidia from the clinical isolate
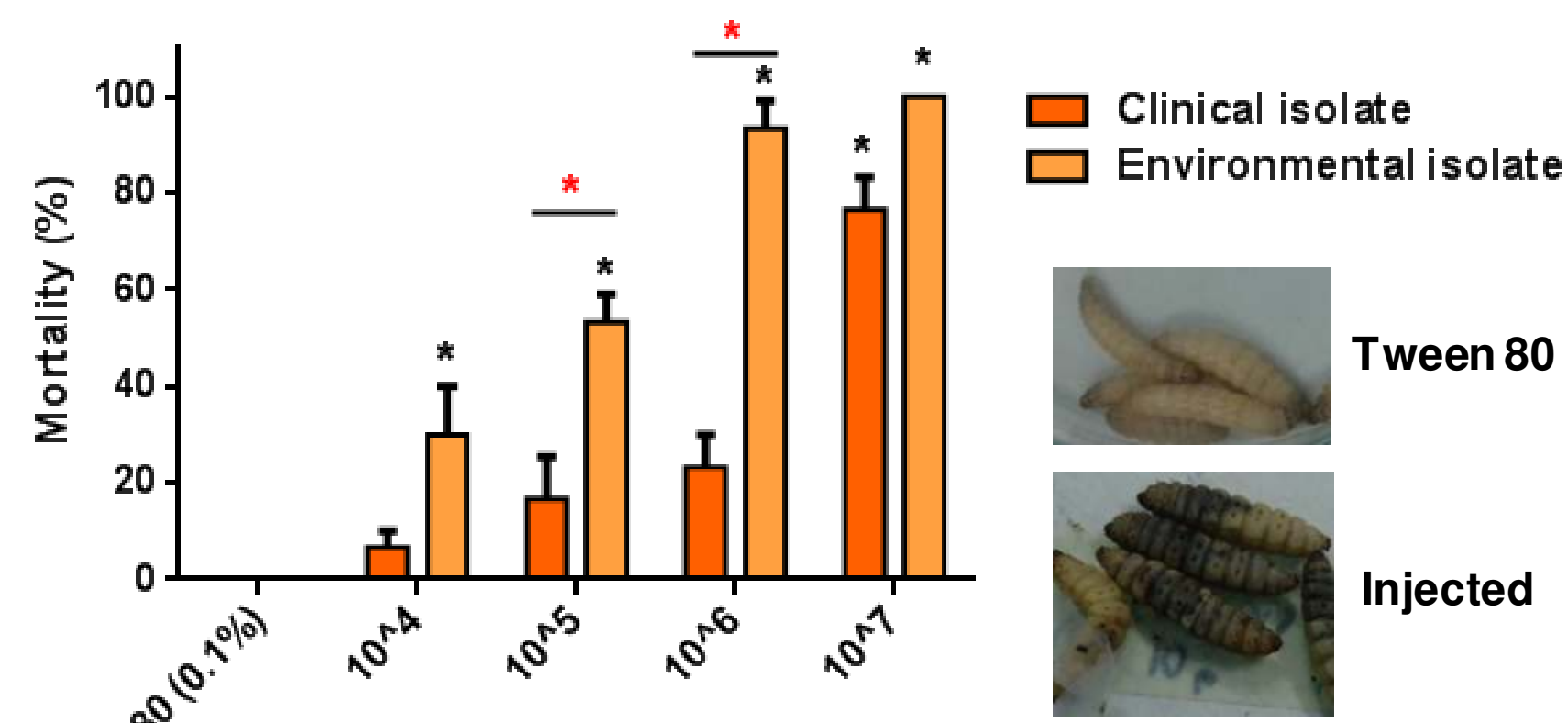

2. Methanol extracts from $F$. oxysporum isolates have different acid methyl esters concentrations.

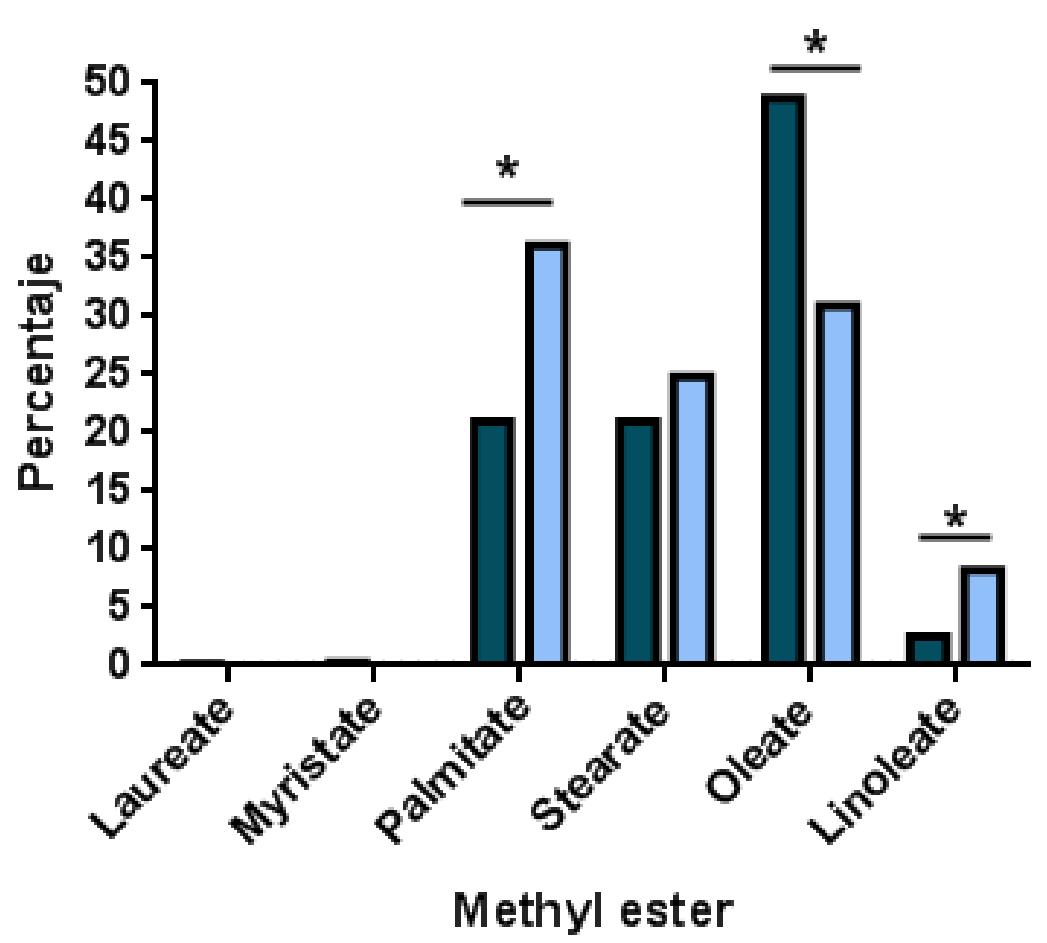

3. Only methanol lipids extracted from $F$. oxysporum environmental isolate caused mortality in caterpillars injected.

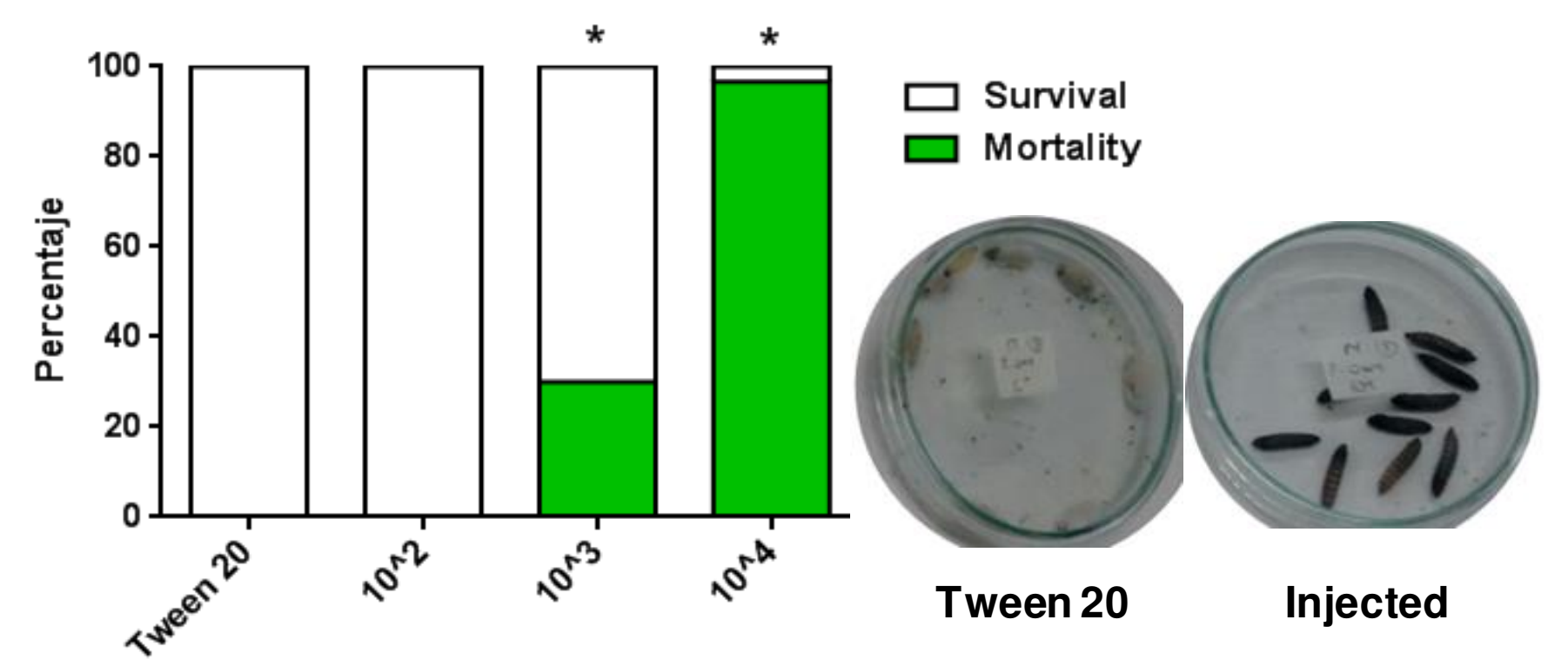

Methanolic lipids $(\mu \mathrm{g} / \mathrm{mL}$ )

4. Co-stimulus of conidia and lipids from the environmental isolate increases the caterpillars mortality compared with individual treatments.

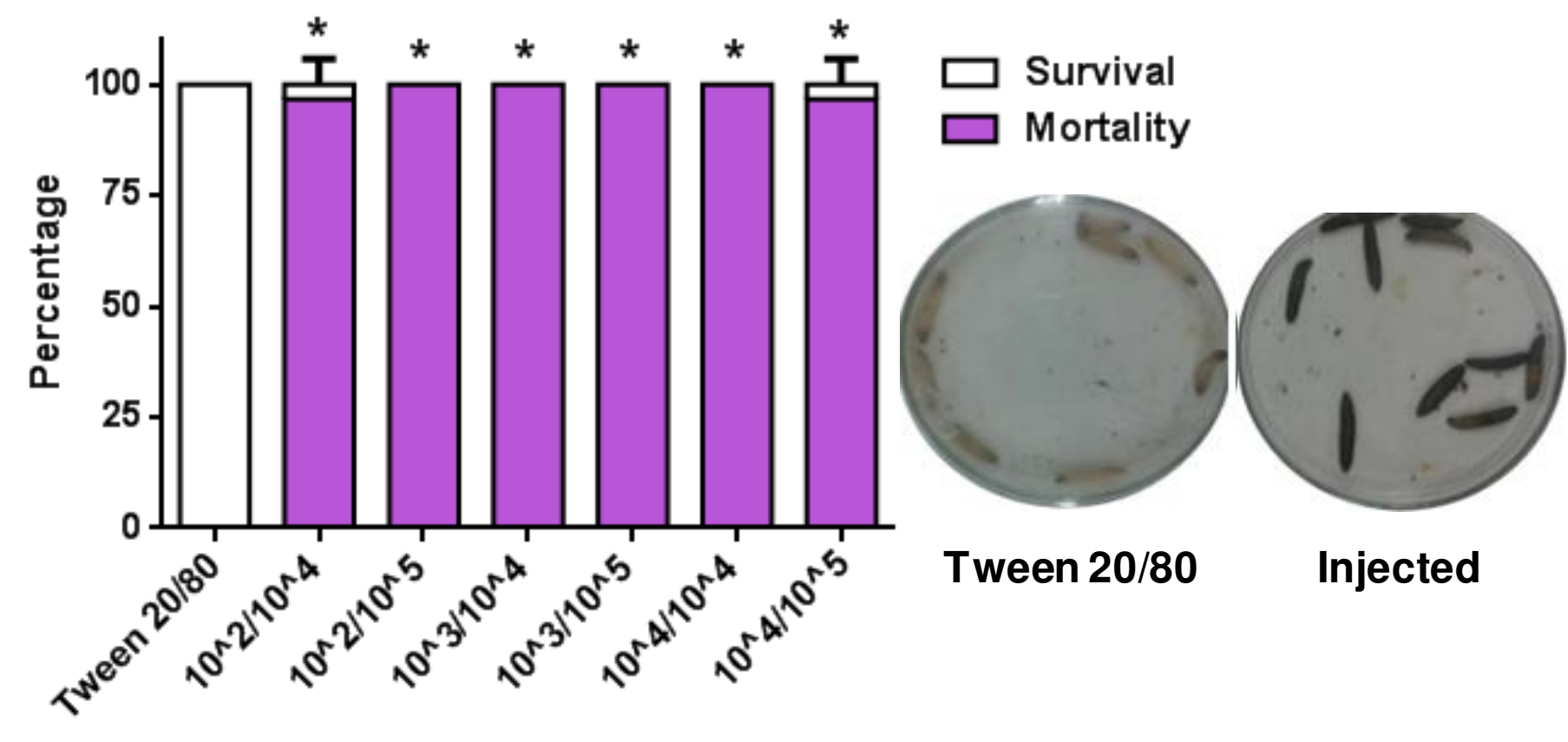

Methanolic lipids concentration / conidia ( $\mu \mathrm{g} / \mathrm{mL} /$ conidia $/ \mathrm{mL}$ )

Concentration (conidia/mL)

\section{CONCLUSIONS}

- Mortality of G. mellonella caterpillars was greater when conidia from F. oxysporum environmental isolate were injected to them.

- Methanol lipids extracted from $F$. oxysporum isolates have different acids methyl esters composition and the lipids extracted from the environmental isolate caused mortality of caterpillars injected.

- The mortality with the co-stimulus of conidia and lipids from the environmental isolate was approximately $100 \%$ compared with individual treatments. 